\title{
PENSER LA CRISE ÉCOLOGIQUE : REPRÉSENTATIONS ET PRATIQUES FRANCO-ALLEMANDES
}

Sabine Caillaud, Nikos Kalampalikis, Uwe Flick

\section{Presses universitaires de Liège | «Les Cahiers Internationaux de Psychologie Sociale »}

2010/3 Numéro 87 | pages 621 à 644

ISSN 0777-0707

Article disponible en ligne à l'adresse :

http://www.cairn.info/revue-les-cahiers-internationaux-de-psychologiesociale-2010-3-page-621.htm

\section{Pour citer cet article :}

Sabine Caillaud et al., «Penser la crise écologique : représentations et pratiques franco-allemandes », Les Cahiers Internationaux de Psychologie Sociale 2010/3 (Numéro 87), p. 621-644.

DOI 10.3917/cips.087.0621

Distribution électronique Cairn.info pour Presses universitaires de Liège.

(c) Presses universitaires de Liège. Tous droits réservés pour tous pays.

La reproduction ou représentation de cet article, notamment par photocopie, n'est autorisée que dans les limites des conditions générales d'utilisation du site ou, le cas échéant, des conditions générales de la licence souscrite par votre établissement. Toute autre reproduction ou représentation, en tout ou partie, sous quelque forme et de quelque manière que ce soit, est interdite sauf accord préalable et écrit de l'éditeur, en dehors des cas prévus par la législation en vigueur en France. Il est précisé que son stockage dans une base de données est également interdit. 


\section{Penser la crise écologique :}

représentations et pratiques francoallemandes

Thinking the ecological crisis: Representations and practices in France and Germany

Sabine CAILLAUD*, Nikos KALAMPALIKIS* et Uwe FLICK**

* Laboratoire " Groupe de Recherche en Psychologie Sociale » (GRePS, EA 4163), Institut de Psychologie, Université de Lyon (Lyon 2), France

** Alice Salomon FH, University of Applied Sciences, Berlin, Allemagne 
Cet article interroge les significations de la crise écologique en France et en Allemagne. Après avoir montré comment I'approche des représentations sociales et l'idée d'une " histoire humaine de la nature " permettent de dépasser certains problèmes inhérents à l'étude de l'objet, nous proposons d'aborder la crise écologique sous l'aspect des motifs liés aux pratiques. Pour cela, nous nous appuyons sur les résultats d'une étude comparative France/Allemagne et plus précisément sur les données issues de la méthode des focus groups. Les résultats montrent la nature antithétique des thêmata à partir desquels la crise écologique est pensée dans les deux pays et leur rapport aux pratiques écologiques associées.
This article examines how the ecological crisis makes sense in French and Germany. First, social representations' approach and the idea of "a human history of nature" help us to overcome some difficulties linked to the object. Then, we propose to approach the ecological crisis by considering motives for ecological practices. We use data from a comparative study in France and Germany and more specifically data from Focus Groups. Results show the antithetic nature of thêmata used to think about the ecological crisis in both countries and their connection with associated ecological practices.

La correspondance pour cet article doit être adressée à Sabine Caillaud, Laboratoire « Groupe de Recherche en Psychologie Sociale », Institut de Psychologie, Université de Lyon, 5 avenue Pierre Mendès France, 69676 Bron CEDEX, France. Courriel : <sabine.caillaud@univ-lyon2.fr>.

Le premier auteur a réalisé les opérations de recherche et d'analyse. Le second et le troisième auteur ont supervisé la phase du recueil respectivement en France et en Allemagne et ont participé au travail de réflexion et d'analyse. 
« Un sentiment d'urgence et une tonalité plus alarmiste que jamais » (Le Monde, 4/12/07) ; " des risques littéralement ingérables entraînant les sociétés dans une spirale incontrôlée de dangers croissants » (Libération, 3/12/07) ; " les scénarios qui se dessinent font aussi peur qu'un film de science-fiction » (Die Welt, 3/12/07) ; " humanité au bord du gouffre » (Frankfurter Rundschau, 12/12/07). C'est en ces termes que des quotidiens français et allemands évoquaient en 2007, à l'occasion d'une conférence de l'ONU sur le climat, les conséquences du changement climatique. La crise climatique, et plus généralement la crise écologique, renvoie ainsi à des risques perçus comme inquiétants et sévères.

La crise écologique à laquelle nos sociétés font face aujourd'hui présente un caractère inhabituel. Les risques sont devenus globaux et les frontières, qui auparavant offraient l'illusion d'une protection, n'ont plus d'effet (Beck, 2001). Tchernobyl a été en ce sens un événement emblématique, soulignant en Allemagne, par exemple, la porosité du rideau de fer (Rudolf, 2005). Le changement climatique est un autre exemple type de risque écologique globalisé. L'apparition du concept scientifique " écosystème » a contribué à une augmentation de traités internationaux concernant l'environnement (Frank, 1997), signe d'une prise en compte globale des risques. L'image de la Terre vue de l'espace a joué un rôle similaire (Acot, 1998).

Dans ce contexte de crise écologique, les pratiques individuelles sont appelées à participer au changement quasiment au même titre que les politiques transnationales. Ainsi, pour ne prendre qu'un exemple relatif au climat, les individus sont invités à préférer le vélo ou les transports en commun à leur voiture, on leur conseille de favoriser une alimentation produite à proximité, ou encore de faire des économies d'énergie. Aussi, cette crise écologique met les individus face à un certain paradoxe : la solution aux risques globaux se situe dans des pratiques quotidiennes et locales. Comment réagissent-ils à ce paradoxe ? Autrement dit, font-ils un lien entre leurs pratiques individuelles et des problèmes écologiques globaux?

La crise écologique constitue ainsi un double enjeu pour la psychologie sociale. Dans un premier temps, si on se situe dans le champ d'étude des représentations sociales, elle constitue un objet aussi global que nouveau avec lequel on cherche à se familiariser, auquel il faut faire face. En ce sens, il est intéressant de comprendre comment la pensée sociale envisage ces différents aspects de la crise écologique et comment les pratiques associées y sont pensées. Dans un second temps, la psychologie sociale peut apporter un certain nombre de solutions visant au changement des pratiques individuelles (Buckhout, 1972). L'objectif de cet article est plus modeste. II s'agira de comprendre les représentations sociales de cette crise à travers les significations accordées aux pratiques écologiques en France et en Allemagne.

\section{La crise écologique : un défi pour la psychologie sociale}

Penser la crise écologique en sciences humaines et sociales, et en particulier en psychologie sociale, n'est pas sans soulever un certain nombre de questions. En particulier, deux problèmes méritent d'être évoqués ici. Un premier questionnement repose sur le dualisme nature/culture qui est le résultat $d^{\prime}$ 'une longue histoire 
des sciences (Lenoble, 1969). Une manière radicale d'illustrer le problème posé par ce dualisme est de considérer que, si on l'accepte, alors le chercheur a le choix entre deux positions (Acot, 1998) : la première est de penser I'Homme comme un être éminemment biologique. Cette perspective rend inintéressante toute étude en sciences sociales sur le rapport Homme-nature, $c^{\prime}$ est aux sciences naturelles de s'en charger. La seconde est de considérer la nature comme ce dont l'Homme est absent, mais cette position ne rend guère plus attrayante la question écologique en sciences sociales. En conséquence, la question de la nature a souvent été considérée par les sociologues comme une question idéologique (Kalaora, 1998) : les écologues qui décrivent les risques deviennent dans ce cadre des individus en quête de légitimité professionnelle et de reconnaissance.

Ce dualisme n'est pas problématique en soi. Mais la crise écologique actuelle fait de la nature un "équipement interne » de notre société (Beck, 2001) et constitue ainsi une sorte de défi pour les sciences humaines et sociales. En effet, il devient intéressant de comprendre : comment les gens pensent-ils la crise écologique ? En quoi constitue-t-elle, ou non, une menace? Et comment les individus y font face?

Un deuxième questionnement concerne plus particulièrement la question des pratiques écologiques. Une question essentielle se pose : qu'est-ce que signifie agir de manière écologique ? Comment définir ce qui est une pratique écologique et ce qui ne l'est pas?

D'aucuns prétendraient qu'il suffirait de prendre pour repères les indications données par différents livrets d'informations et de sensibilisation comme par exemple "Le Petit Livre Vert pour la Terre » publié par l'Agence de l'environnement et de la maîtrise de l'énergie (ADEME) en 2005. Mais cela signifie en premier lieu que I'on accepte, et que l'on partage, la définition de ce qu'est l'écologie pour l'ADEME. De plus, certains paradoxes apparaissent dans la liste même des gestes conseillés. Ainsi, pour ne prendre qu'un exemple relativement simple, il est conseillé de trier ses déchets et en même temps il est conseillé d'éviter les produits jetables. Peut-on considérer que ces deux gestes différents soient comparables ? Leur effet n'est pas le même : trier devrait être, selon ce guide, la dernière solution à envisager. Par ailleurs, ces deux comportements ne sont pas influencés par les mêmes facteurs individuels (Barr, 2007).

Mais une question encore plus importante que le degré écologique de chaque pratique se pose. Il s'agit du sens même que l'individu donne à un comportement. En effet, l'achat de produits "bio » est souvent utilisé pour opérationnaliser les comportements écologiques (Olli, Grendstad et Wollebaek, 2001 ; Thogersen et Ölander, 2006), or la consommation de produits issus de l'agriculture biologique est aujourd'hui considérée, non plus seulement comme une pratique de protection de la nature, mais aussi comme une manière de protéger sa santé. Par ailleurs, certaines études opérationnalisent le comportement de tri par le poids des déchets (Matheau-Police et Moser, 2002 ; Kurz, Linden et Sheehy, 2007). Elles se confrontent à une limite importante qu'elles ne manquent pas d'ailleurs de souligner : comment être certains que la différence de poids des déchets triés n'est due qu'au 
comportement de tri ? Effectivement, certaines personnes peuvent être sensibilisées à l'écologie au point de réduire leurs déchets, d'autres, ayant des revenus plus faibles, achèteront moins de journaux et donc en jetteront moins. Sont-ils moins " écolos » pour autant?

Le fait de s'intéresser à des comportements effectifs plutôt qu'à des comportements auto-déclarés présente, certes, de nombreux avantages mais n'est pas non plus sans poser questions. Pour prendre un autre exemple, peut-on considérer comme une pratique écologique le fait d'utiliser des ampoules basse-consommation pour diminuer sa facture d'électricité ? On le voit, la question de la définition des pratiques écologiques n'est pas aussi simple à résoudre que le laisse croire une lecture superficielle des "petits guides verts".

Ces deux problèmes étant posés, nous allons nous attacher à montrer comment I'approche des représentations sociales (RS) permet d'y répondre.

\subsection{L'histoire humaine de la nature et l'approche des représentations sociales}

L'idée « d'une histoire humaine de la nature » est de ces notions évidentes et tout à la fois complexes. Évidente, car elle considère que la nature « $C^{\prime}$ est l'Homme avec la matière » (Moscovici 1968, p. 36). Mais complexe aussi, dans la mesure où cette perspective nous engage à considérer que le dualisme nature/culture est le résultat $d^{\prime}$ 'une construction historique de l'objet nature dans le temps. Ainsi, les Hommes, dans leur rapport à d'autres Hommes, construisent l'objet nature. Nature et culture, ou bien nature et société, ne peuvent donc être pensées l'une sans l'autre. Elles sont dans une relation dialectique : "En agissant sur la matière, I'Homme agit simultanément sur lui-même, toute réorganisation des rapports avec les puissances du milieu réel est une réorganisation de ses rapports avec lui-même » (Moscovici, 1974, p. 176). Ainsi, les sociétés choisissent et définissent leur " nature » en établissant des relations avec ce qui les entoure. En ce sens, on peut parler d'une " histoire humaine de la nature ». La nature est donc le résultat d'une pratique et d'une pensée humaine qui auraient pu être différentes (Eder, 1988).

À notre connaissance, seule Gervais (1997) a proposé, à travers une recherche empirique, un rapprochement entre l'approche des RS, telle qu'elle a été développée par Moscovici $(1961,2001)$, et l'idée d'une histoire humaine de la nature (Moscovici, 1968). Elle envisage les différents «états de la nature » décrits par Moscovici (1968) comme des RS de la nature. Selon nous, ce parallèle risque de porter à confusion : les RS sont spécifiques aux sociétés modernes, ce n'est pas le cas des " états de la nature ». Mais un autre lien peut être proposé entre ces différents travaux, si l'on s'attache à comprendre leur proximité épistémologique.

Dans les deux approches (RS et histoire humaine de la nature), il s'agit de considérer que la réalité se construit sans cesse, de manière dialogique, même si le changement n'est pas toujours perceptible (Markova, 2007). Précisons notre pensée. II est assez simple d'accepter l'idée selon laquelle les RS de la nature se construisent au sein d'une triade Ego-Alter-Objet ${ }^{1}$. Ainsi, la surface du triangle composé d'Al- 
ter-Ego-Objet devient un rapport à la nature. Si I'on ajoute à présent la dimension temporelle, alors on retrouve le modèle du Toblerone proposé par Bauer et Gaskell $(1999)^{2}$. En effet, les choses ne sont jamais posées, elles évoluent sans cesse. Le modèle reprend donc cette même triade et montre comment la surface représentant le rapport des Hommes à la nature évolue dans le temps. L'espace intérieur du modèle, c'est l'histoire humaine de la nature. Ainsi, et c'est là l'essentiel de notre propos, I'approche des RS, de par sa proximité avec la notion d'histoire humaine de la nature, permet de considérer que le dualisme nature/culture est un rapport contextuellement et historiquement situé.

Aborder la crise écologique par I'approche des RS permet donc de l'envisager comme un objet socialement construit. Par exemple, on ne considère plus, dès lors, que les agriculteurs soient responsables de la crise écologique, mais c'est la forme sociale spécifique d'une catégorisation naturelle qui les obligerait à des pratiques désignées défavorables à la nature (Eder, 1988). Enfin, cette approche permet aussi de réhabiliter la pensée sociale pour l'envisager autrement que sous l'aspect d'un écart entre savoir scientifique et savoir profane ${ }^{3}$.

Dans ce cadre, la notion de thêmata, définie comme des idées sources (Moscovici et Vignaux, 1994), comme des taxonomies de nature oppositionnelle capables de générer des RS (Markova, 2007), devient pertinente pour tenir compte justement de l'aspect dialogique des RS.

\subsection{Le lien entre représentations sociales et pratiques}

Revenons maintenant au problème posé par la définition des pratiques écologiques. Une grande partie de la littérature en psychologie sociale s'intéresse aux comportements des individus et non pas à leurs actions. La distinction entre les deux mérite d'être brièvement rappelée. Il s'agit du caractère intentionnel de l'action. Autrement dit, l'acteur poursuit un but : celui de changer son environnement ou d'en empêcher le changement (Cranach et al., 1982). L'acteur cherche donc à intervenir sur le cours normal des événements pour obtenir un résultat. Une même intention peut être réalisée par des pratiques différentes (Léont'ev, 1977). Un même comportement peut également renvoyer à des intentions différentes (pour les ampoules basse consommation, par exemple : faire des économies ou protéger la nature). Enfin, I'action dépend dans une certaine mesure du contexte et du système dans lequel est placé l'acteur, ce dernier disposant néanmoins d'une marge de liberté (Crozier et Friedberg, 1977). Considérer que l'acteur agit selon un but n'implique pas de considérer qu'il s'agisse nécessairement d'un intérêt économique (cf. la notion de capital symbolique chez Bourdieu, 1994). Caillé (2009), quant à lui, élabore une véritable théorie des motifs de l'action fonctionnant de manière dialogique et plaçant l'acteur dans des relations interpersonnelles complexes, nous y reviendrons.

Comprendre et étudier les pratiques écologiques implique d'accorder une importance aux intentions, aux motifs des acteurs, aux sens qu'ils donnent à leurs pratiques. Comment I'approche des RS permet-elle de prendre en compte cet aspect des pratiques? 
Le lien entre RS et pratiques peut être envisagé selon différentes perspectives. Abric (2003) considère ce lien de manière dialectique, autrement dit, les représentations déterminent les pratiques, et les pratiques peuvent également être à l'origine du changement des RS, si la situation est perçue comme irréversible (Flament, 1987). Cela nous amène à faire un premier constat et à soulever un paradoxe (Rouquette, 2000) : d'une part, les RS guident l'action, et dans ce cas l'action confirme les représentations. D'autre part, seule l'action qui les infirme est éventuellement celle qui transforme les RS. Comment et pourquoi une action contradictoire peut-elle avoir lieu si les représentations servent de guide à l'action? Ce sont des éléments extérieurs qui vont inciter à une action nouvelle (Rouquette, 2000). Toutefois, on peut se demander dans quelle mesure cet événement extérieur ne va pas tout d'abord faire changer la représentation qui va donner lieu, alors, à une action inhabituelle.

Une autre manière d'envisager le lien entre RS et pratiques a été développée dans la monographie classique de Jodelet (1989) amenant Moscovici (1989, p. 26), lors de sa préface, à qualifier "d'actions représentationnelles » des conduites qui ne sont pas déterminées "par des causes au sens strict du mot, ni par de simples contraintes, mais par des intentions ». Selon Wagner (1994), de nombreuses études sur les RS envisagent les pratiques selon un modèle de causalité issu de la folk psychology, or, "les RS n'ont pas été conçues au départ comme des cognitions intervenant entre le stimulus et le comportement mais comme des structures symboliques qui relient les deux » (op. cit., p. 244, notre traduction).

Cette façon d'envisager le lien entre RS et pratiques retiendra notre attention dans le cadre précis de cette étude dans la mesure où elle met l'accent sur l'intention et les motifs des acteurs, autrement dit sur le sens des pratiques. Cette perspective permet d'envisager la crise écologique sous un angle nouveau, ou tout du moins inhabituel : quelles RS de la crise écologique peut-on mettre en évidence à travers les actions quotidiennes?

\section{La comparaison France/Allemagne}

Une seconde spécificité de notre étude réside dans la comparaison France/Allemagne que nous avons mise en place pour mener notre recherche. II n'est plus nécessaire de souligner l'importance du contexte culturel en psychologie sociale (cf. Faucheux, 1976 ; Jodelet, 2002, 2006). En effet, les processus de formation, transformation, reconstruction des RS sont des processus cognitifs et sociaux. Autrement dit, ces processus dépendent bien du contexte socioculturel dans lequel est familiarisé un objet. La comparaison entre divers contextes s'avère alors pertinente pour éclairer le fonctionnement de ces processus et pour expliquer les différences de contenu qui en résultent (Flick, 1996). Aussi, la comparaison est utilisée ici dans le but de comprendre, d'expliquer, de mettre en évidence des régularités sociales afin de développer une théorie plus générale (Vigour, 2005).

Différentes raisons expliquent le choix de la France et de l'Allemagne. Ces deux pays présentent un certain nombre de similarités (deux pays européens modernes, démocratiques, industriels et capitalistes, avec des populations et une structure éco- 
nomique comparables). Il s'agit donc ici de comparer des systèmes dits très similaires (Vigour, 2005). Toutefois, ces mêmes pays présentent des différences importantes quant à l'objet écologie du point de vue de sa traduction idéologique et politique que nous allons tenter de présenter brièvement (l'espace de cet article ne permettant pas d'approfondir le détail).

En France et en Allemagne (de l'Ouest ${ }^{4}$ ), on voit fleurir dans les années soixante différentes associations vertes dont les parcours seront différents. En Allemagne, l'ensemble de ces associations, défendant des intérêts locaux (Bürgerinitiativen), se regroupe peu à peu autour d'un discours idéologique commun et fédérateur (Jacquiot, 2007), qui s'ancre autour d'une réflexion éthique (Chibret, 1991). En France, au contraire, ces associations écologistes s'ancrent dans le mouvement politique contestataire de mai 68. Mais cet ancrage politique ne rassemble pas l'ensemble des associations et certaines considèrent que l'écologie doit rester en dehors de tout débat politique et doit se référer uniquement à la science pour décider de ses actions (Chibret, 1991). Le mouvement Vert français est alors divisé. Ce destin différent des associations écologistes en France et en Allemagne reste inexpliqué. L'hypothèse d'une Allemagne marquée par son passé nazi et cherchant à se déculpabiliser est rejetée au profit d'explications plus sociologiques (Jacquiot, 2008). D'autres explications reposent sur un héritage plus ancien : les courants philosophiques des Lumières en France et des Romantiques en Allemagne marqueraient aujourd'hui encore des rapports différents à la nature (Ferry, 1992). Le recours au savoir scientifique comme seul référent dans de nombreuses associations en France fait d'ailleurs écho à la tradition des Lumières (Ollitrault, 2001).

Il est probable que les courants philosophiques qui ont marqué ces deux pays de manière différenciée aient laissé des traces (jardin à la française, jardin public sauvage en Allemagne ${ }^{5}$ ). Mais il est également probable que ces éléments du passé aient fait l'objet d'utilisations multiples dans le présent (Haas et Jodelet, 1999). Ainsi, Eder (2000) considère que l'écologie a pu constituer pour l'Allemagne d'après-guerre un objectif fédérateur et qu'elle aurait à cet effet réutilisé certains éléments de son passé (notamment le courant Romantique). II n'en reste pas moins que des études plus récentes montrent que des différences quantitatives persistent entre les Français et les Allemands, tant au niveau de leurs attitudes que de leurs comportements écologiques (p.ex. Eurobaromètre, 2002 ${ }^{6}, 2004^{7}$ ). De même, des études sociologiques soulignent des différences importantes, que cela soit chez les éco-conseillers (Rudolf, 1998) ou au sein du discours public sur certaines questions environnementales telles que les déchets (Keller, 1998).

Dans ces deux contextes différents, quels sens sont accordés aux pratiques écologiques par les acteurs eux-mêmes? Qu'est-ce que cela nous dit de la manière dont la crise écologique y est pensée?

\section{Méthode}

La recherche que nous avons menée sur les RS de l'écologie en France et en Allemagne s'appuie sur un plan méthodologique reposant sur le principe de la triangu- 
lation des méthodes (Flick, 1992). Pour cela, des entretiens individuels, une analyse de presse et des focus groups ont été réalisés. Ici, c'est une partie des données issues des focus groups qui va illustrer notre propos.

\subsection{Interaction et structuration}

On peut définir les focus groups comme des « espaces de communication permettant d'engager, d'observer et d'analyser des interactions, des souvenirs et des représentations dans I'action » (Kalampalikis, 2004, p. 282). Ils se distinguent principalement des autres méthodes de recueil de données par l'utilisation de l'interaction (Kitzinger, 1994). Le contexte du groupe, ses effets d'influence (consensus, normalisation, polarisation), ne sont pas ici un biais pour saisir l'opinion individuelle, mais ils deviennent significatifs en soi pour la recherche. C'est en s'intéressant aux processus d'interaction du groupe que cette méthode est exploitée pleinement (Kitzinger, 1994). Il peut même être parfois plus pertinent de comparer les groupes, non pas selon les opinions qui se dégagent, mais selon les différents processus par lesquels ces groupes sont passés : y a-t-il débat autour d'une opinion ? est-ce qu'un consensus se forme ? Comment ? etc. (Barbour, 2008). Concernant notre objet, la crise écologique, I'utilisation des focus groups est d'autant plus adaptée qu'il s'agit d'un objet social, abordé lors de communications aussi bien médiatiques qu'interpersonnelles. Enfin, interroger des sujets sur leurs pratiques écologiques en focus groups permet de tenir compte du caractère paradoxal de ces pratiques : la somme de pratiques locales considérée comme solution possible à une crise globale.

Cependant, bien que la situation du focus group rappelle une situation naturelle de discussion, elle est générée par le chercheur (Kitzinger, Markova, Kalampalikis, 2004). Pour composer avec cet artefact et rendre la discussion la plus spontanée possible, Kalampalikis $(2002,2007)$ revisitant les premiers usages du focus group par Merton, propose de la structurer. Pour cela différents supports/stimuli peuvent être utilisés (photographies, extraits de texte, sondages, etc.) afin de rythmer la discussion, susciter et renouveler l'intérêt des participants, maintenir la focalisation sur les dimensions thématiques à explorer, structurer l'analyse. Par ailleurs, ils fournissent des informations sur les situations à partir desquelles les participants co-construisent des significations.

En ce sens, différents supports ont été proposés durant les focus groups que nous avons menés. Par exemple, après avoir discuté autour d'une carte sur la production des gaz à effet de serre, le groupe était invité à dresser une liste de leurs pratiques écologiques et d'en évaluer sur une échelle de 1 à 4 le degré d'efficacité ${ }^{8}$. Un des participants notait les pratiques et cochait les réponses dans un tableau préalablement préparé. Enfin, les choix de modération de la dynamique du groupe visaient à favoriser la discussion, l'échange entre les participants, les débats... Nous intervenions donc afin de favoriser au maximum l'interaction, pour inviter à poursuivre un débat qui semblait être laissé de côté, pour remettre en cause les " taken for granted », pour discuter les paradoxes (Kitzinger, 1994). 


\subsection{Population et procédure de réalisation}

L'ensemble des participants est âgé de 28 à 40 ans. Cette classe d'âge a été retenue dans la mesure où on y trouve des rapports suffisamment hétérogènes à la nature et à l'écologie (Schuster, 2003). On s'assure ainsi d'une certaine diversité des données recueillies. La taille des groupes a été déterminée de manière à ce que le débat soit possible, que l'ensemble des participants puissent s'exprimer et que la retranscription reste plausible. Aussi, le nombre de participants a été fixé entre 4 et 5 personnes. Certains participants se connaissaient, d'autres non ${ }^{9}$.

Dix focus groups ont été conduits : 4 en France (Lyon), et 6 en Allemagne, (Berlin) ${ }^{10}$. 41 Français et Allemands ont participé à ces focus groups. Le lieu de la discussion était en général chez un des participants, néanmoins pour des questions d'accessibilité certaines ont été menées à Berlin au sein des locaux d'une université.

L'ensemble des discussions a été enregistré et retranscrit. L'analyse s'est déroulée en différentes étapes. En premier lieu, nous avons dressé la liste des thèmes successifs abordés par les participants. Cette étape constitue le point de départ d'une analyse de contenu, elle présente l'avantage de fournir une bonne représentation des thématiques du corpus et de pouvoir s'y repérer facilement (Markova, 2003 ; Flick, 2008). Un index des thèmes est ainsi obtenu et sera présenté.

Dans un second temps, nous avons engagé une analyse dialogique des focus groups en prenant en compte le contenu et la dynamique du groupe. Pour cela, nous nous sommes appuyés sur les outils analytiques proposés dans la littérature sur les focus groups (cf. Stewart, Shamdasani, Rook, 2007 ; Kalampalikis, 2004 ; Morgan, 1996) afin d'observer comment les thèmes dégagés s'articulent selon la focalisation sur les supports proposés et l'interaction des participants. Le niveau d'analyse était double : le cheminement du groupe (consensus, formation de normes, minorité) et le cheminement de chaque participant (changement d'opinion...) ont été pris en compte. Ces deux étapes de l'analyse (thématique et dialogique) ne sont pas sans rappeler les étapes définies pour la recherche de thêmata (Moscovici et Vignaux, 1994).

\section{Résultats : des tensions structurant le discours}

\subsection{Description du contenu des discussions}

Un premier résultat concerne les pratiques qui ont été discutées en France et en Allemagne. Le tableau ci-dessous en donne un aperçu, ainsi que la fréquence à laquelle chaque pratique a été évoquée. Il s'agit des pratiques qui figurent sur les listes construites lors de la discussion. Etant donné que le nombre total de focus groups est de 10, et que certaines pratiques sont courantes en Allemagne et infaisables ou peu développées en France (consigne, choix du fournisseur électrique), il est difficile d'établir avec certitude des conclusions. Néanmoins certaines remarques peuvent être faites.

Certaines pratiques sont très fréquemment citées dans les deux pays, c'est le cas du tri et du choix des modes de transport. L'achat de produits biologiques est également 
Tableau 1 : Pratiques discutées et fréquence d'évocation en France et en Allemagne

\begin{tabular}{ccc}
\hline & \multicolumn{2}{c}{ Fréquence $(\%)$} \\
\hline Pratiques écologiques mentionnées & Allemagne & France \\
\hline Tri & 100 & 100 \\
Transports & 66 & 50 \\
Réduction déchets & 83 & 100 \\
Ne pas utiliser de sac plastique & 33 & 75 \\
Economie énergie & 16 & 0 \\
Energies alternatives & 100 & 25 \\
Consignes & 33 & 50 \\
Achat saison, local & 16 & 0 \\
Pas d'avion & 50 & 100 \\
Achat bio & 33 & 75 \\
Manger végétarien & 83 & 75 \\
Fournisseur électrique & 66 & 25 \\
Pas de spray pour CFC & 33 & 0 \\
Fumer & 16 & 0 \\
Action sociale & 16 & 0 \\
\hline & 50 \\
\hline
\end{tabular}

assez fréquemment débattu. En revanche, certaines différences sont assez surprenantes, comme la plus grande importance accordée en France à la réduction des déchets ou aux achats de produits de saison produits localement, ou en Allemagne aux économies d'énergie et à une alimentation plus végétarienne. Ces quelques différences indiquent que les groupes des deux pays semblent se distinguer quant à l'importance accordée à certaines pratiques. Enfin, de manière générale, les pratiques sont évaluées comme très efficaces ou efficaces.

Le tableau ci-dessous donne à présent l'index des thèmes abordés durant les discussions. Comme nous l'avons souligné, il s'agit ici d'une analyse thématique de contenu qui ne prend pas en compte les interactions.

La première colonne de ce tableau montre quels sont les thèmes généraux développés lors des discussions. Les colonnes suivantes montrent comment chacun de ces thèmes est abordé de manière concrète dans les groupes de chaque pays.

D'ores et déjà, on peut souligner que les participants ne se contentent pas d'évaluer les pratiques en fonction d'une causalité scientifique, en fonction de critères logico- 


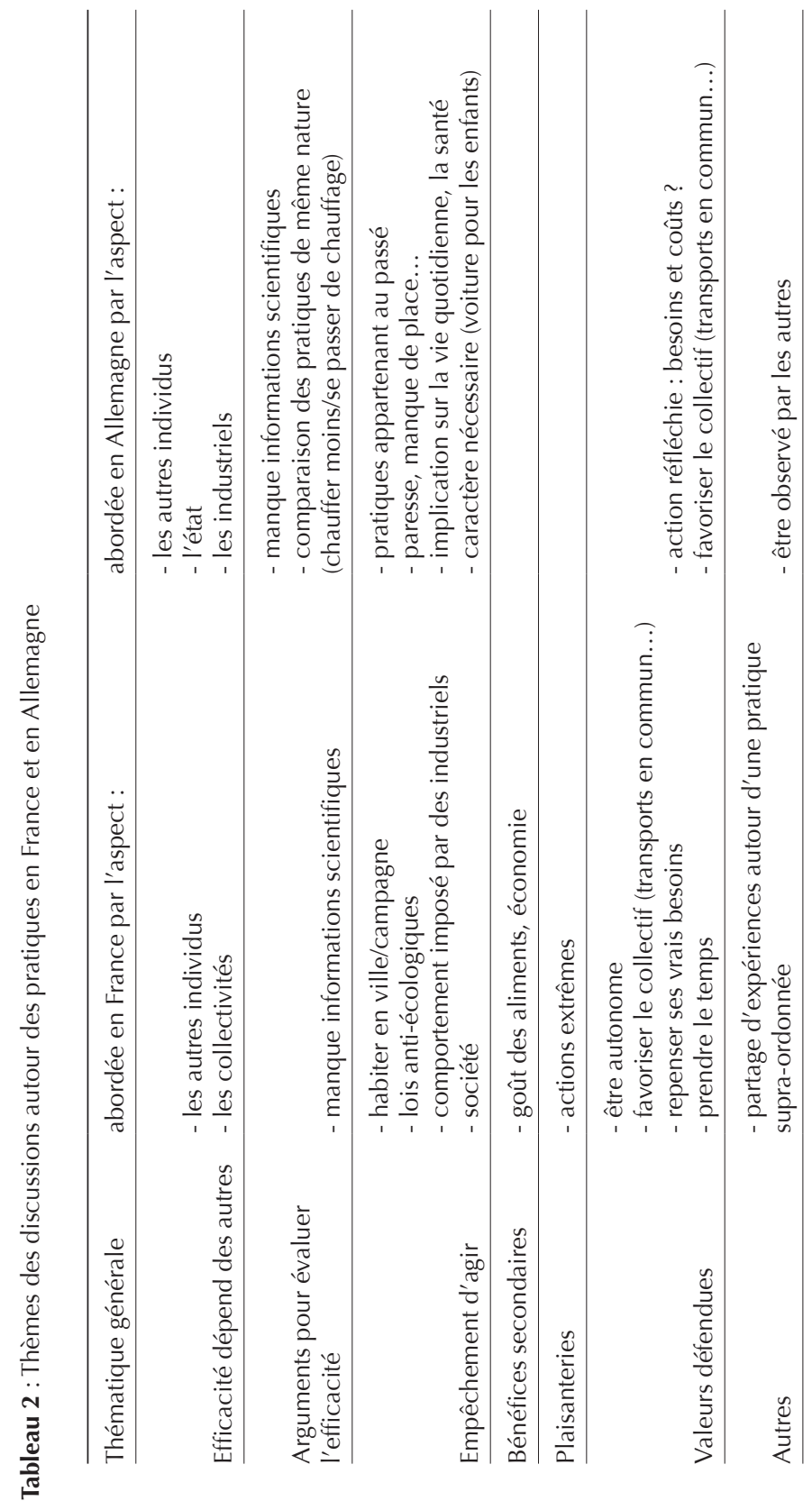


déductifs. D'autres éléments sont pris en compte, comme les bénéfices secondaires ou le comportement des autres. Mais deux autres thèmes retiennent également notre attention : ce qui empêche d'agir et les valeurs défendues. Ces deux thèmes sont abordés de manière différente en France et en Allemagne.

La suite de nos résultats va montrer comment ces thèmes s'articulent à la dynamique de la discussion.

\subsection{L'importance des autres et le rôle de la majorité}

Les groupes ont peu recours à des critères scientifiques, souvent jugés incertains ${ }^{11}$, pour évaluer leur pratique. Comment alors s'y prennent-ils?

Prenons l'exemple du FG F1, où les participants discutent des produits de nettoyage écologique :

\section{FG F1}

J : que l'on ait des, on ait des indices. Qui objectivement soient capables de nous dire voilà dans votre quotidien si vous faîtes ça, la répercussion elle sera ça. Des indices objectifs et pas de vendeurs d'assouplissant biologique tu vois ce que je veux dire, parce que c'est ça le, c'est très tendance très biaisé, des vrais objectifs avec des études scientifiques

Ch : ouais moi je suis d'accord

$S$ : moi je suis d'accord mais je suis contente de me dire ce que j'achète c'est propre

$\mathrm{J}$ : moi j'ai pas une confirmation scientifique ça marche, ça marche pas

Ch : ça n'a pas de valeur

$\mathrm{S}$ : beh moi je pense que si tout le monde se met à laver tu vois en machine de vaisselle et de linge moi ce que je fais, j'en fais une par jour minimum

Ch : ah ouais

$\mathrm{S}$ : beh si tout le monde fait ça, on va loin quoi. Non mais si tu mets des produits dégueulasses dans ta machine enfin tu vois

Dans cet extrait, S ignore l'absence de certitudes scientifiques avancée par J et s'appuie sur la règle de la majorité : «si tout le monde le fait » remplace la causalité scientifique incertaine. Et le reste du groupe finit par être d'accord.

Cette manière d'évaluer les pratiques renvoie clairement à l'idée que ces dernières sont efficaces si la majorité des gens agissent ainsi. Le thème "efficacité dépend des autres » (tableau 2) se retrouve dans la dynamique même du groupe. Observons I'extrait ci-dessous ${ }^{12}$ :

\section{FG D5}

$M$ : faire des dons pour l'environnement ?... est-ce que ça apporte quelque chose, est-ce que vous le faites?

An : on le fait

$M$ : on le fait hein

$F: c^{\prime}$ est efficace voire très efficace

Ach : est-ce que je l'ai déjà fait?

$M$ : on est déjà trois tu peux faire ce que tu veux $c^{\prime}$ est efficace ça a un résultat 
M souligne ici qu'indépendamment de la réponse de Ach, réaliser à plusieurs (trois) la même pratique amène un résultat. Ainsi, le thème de " l'efficacité qui dépend des autres » se trouve associé à une forme de conformisme. Il est ainsi surprenant de constater qu'un seul des dix groupes ait fait le choix de cocher dans le tableau la réponse de chaque participant. Tous les autres ont cherché à proposer une réponse, celle de la majorité, alors que l'objectif n'était pas d'arriver à un consensus.

Le désaccord au sein des groupes est même assez mal vécu et conduit les participants à des stratégies surprenantes. Par exemple, dans le FG F4, la personne qui notait les réponses du groupe inscrivait ainsi les croix dans des extrémités de la case pour faire ressortir son opinion personnelle, nous signalant au passage de faire attention à la position de la croix. Dans le FG D6, les trois participantes qui utilisent des produits d'entretien écologiques sont confrontées à l'opinion de J qui les considère comme inefficaces. A la fin, la participante (qui a le crayon en main) décide de mettre J à part dans la grille, et le représente à l'aide d'une petite croix, tandis que l'avis de la majorité est représentée avec une grande croix. Par cette stratégie, le groupe reconnait le désaccord, mais il nous signifie où se situe la " grande » majorité.

Que se passe-t-il à présent dans les cas où la majorité reconnait qu'une pratique est inefficace? Les autres participants changent-ils alors d'avis aussi ? Dans le FG F3, un des sujets, travaillant dans une épicerie bio, apprend avec déception que I'agriculture biologique n'est pas systématiquement mieux que l'usage de pesticides pour la qualité du sol. Elle réagit :

\section{FG F3}

So : mais moi je vais aller leur dire au travail demain je vais leur dire mais c'est quoi cette histoire (elle rit) je vais plus manger bio, je vais dire à mes clients arrêtez de manger bio ça sert à rien.

Dans ce type de situation, le participant se met alors à douter de ses propres pratiques et envisage d'en changer. Ce phénomène est d'autant plus surprenant que le groupe de discussion est éphémère. Même si notre méthode ne nous permet pas de savoir si ce changement a eu lieu, ces résultats soulignent l'importance des représentations et du comportement des autres dans I'évaluation des pratiques écologiques, ainsi que la nécessité perçue de partager la même représentation. A présent, nous allons nous intéresser plus spécifiquement à la manière dont les autres thèmes du tableau 2 sont mobilisés dans les discussions.

\subsection{Une évaluation pragmatique pour un ancrage moral en Allemagne}

Comme l'indique le tableau 2, les Allemands ont discuté des pratiques écologiques en fonction de critères pratiques (voir le thème « empêchement d'agir »). Ce critère devient même déterminant pour l'évaluation de l'efficacité des pratiques. La question de la faisabilité des pratiques remplace alors celle de leur efficacité. Dans cet extrait, qui vient illustrer ce thème, l'usage de la voiture est discuté : 


\section{FG D1}

$M$ : enfin je n'aurais pas de voiture si je n'avais pas d'enfant

Su : c'est ce que j'allais dire

$M: c^{\prime}$ est juste à cause des enfants alors je me dis quelle somme d'argent finalement, $\mathrm{d}^{\prime}$ abord l'argent après pour l'environnement, et on se dit c'est vraiment n'importe quoi on a vraiment juste besoin d'une voiture pour les enfants, pour que l'on soit rapidement chez le pédiatre, que l'on soit rapidement à l'école, si on se levait une demi heure plus tôt (elle rit)

Su : c'est vrai mais avec un temps pareil (il pleut fort ce jour là)

Ainsi, les sujets allemands envisagent chaque pratique sous I'angle des implications concrètes sur leur vie quotidienne. Se passer de voiture signifie moins de confort, se lever plus tôt, passer du temps sous la pluie. De même, on accepte certaines concessions pour le quotidien mais pas pour les vacances, etc. Cet aspect de la discussion des groupes nous amène à poser la question suivante : pourquoi accepte-t-on des concessions? Qu'est-ce qui pousse les Allemands à agir de façon écologique? Considérons l'extrait suivant où les participants discutent du tri du verre $^{13}$ :

\section{FG D5}

Ach : je l'ai vraiment vu, j'ai pensé ce n'est pas possible, ils se foutent de moi ou quoi, ils sont venus avec le camion, montent le truc (le container) l'ouvrent, hop et là c'est tombé j'ai dit hein quoi

$\mathrm{F}$ : tout dans le même ou quoi

$M$ : oui évidemment

Ach : oui évidemment ils ouvrent simplement le couvercle et tout est tombé comme ça

$\mathrm{F}:$ il n'y a pas trois compartiments dans le camion?

Ach : non ça s'est simplement ouvert et tout est tombé

$M$ : ils retrient ensuite (ils rient)

An : c'est les boulots à un euro ils trient les débris

Ach : en tous les cas je me disais c'est pas possible, peut-être qu'ils avaient un vieux camion qui ne pouvait pas je sais pas en tous les cas je me sentais un peu.

On peut s'intéresser à la fonction de cette histoire dans la dynamique de la discussion (Barbour, 2008). Le participant vient souligner le sentiment désagréable d'être trompé, qu'on se moque de lui. De même, le parallèle des autres sujets avec les « jobs à un euro », mesure politique très controversée en Allemagne, confirme ce sentiment. On retrouve dans d'autres groupes cette idée d'honnêteté/malhonnêteté :

\section{FG D6}

E : et je trouve que c'est aussi un bon sentiment de pouvoir faire quelque chose soimême, bien que ce soit une goutte d'eau

$\mathrm{J}: \mathrm{c}^{\prime}$ est le sentiment d'être honnête avant tout

E : oui tout simplement

$\mathrm{J}$ : oui que $\mathrm{c}^{\prime}$ est non seulement un engagement politique mais un engagement personnel 
E : oui exactement et que l'on ne renonce pas en disant un truc du style je peux rien y changer, et euh alors je ne trie pas mes déchets non plus, mais parce que c'est remis ensemble après parce que $c^{\prime}$ est ce qui se passe, bon mais tout de même c'est mon petit apport.

Dans un autre groupe, un parallèle est fait entre les pratiques écologiques et les œuvres caritatives :
FG D4
$S:$ je trouve que l'on fait déjà beaucoup
$\mathrm{D}$ : à Noël un don...
C : c'est clair...
$\mathrm{D}$ : comme ça aussi on a à peu près bonne conscience

À travers ces extraits, on voit que les discussions prennent en compte la dimension morale des pratiques écologiques de manière dialogique (moral/immoral). Cette dimension nous permet de comprendre que les pratiques écologiques sont prises dans une sorte de tension entre un intérêt pour soi, immoral (égoïsme) et un intérêt plus moral (empathie, altruisme), dirigé vers un Autre. La triangulation des méthodes nous a permis de montrer que l'Autre est multiple : il peut s'agir d'une personne, d'un environnement, des générations futures, des pays pauvres... Dans le dernier extrait cité, cet aspect moral renvoie même à une dimension religieuse (un don à Noël). Ainsi, c'est dans cette tension que l'on peut saisir le sens des valeurs défendues en Allemagne (voir tableau 2) : penser chaque action en fonction de leur coût pour l'environnement et ses propres besoins.

\subsection{Afficher ses valeurs : des pratiques symboliques en France}

C'est un discours très différent que l'on trouve en France dans les discussions de groupe.

\section{FG F3}

$\mathrm{J}$ : moi je fais du compostage...

Se : ah beh elle habite à la campagne

$\mathrm{J}$ : oui c'est pour ça je peux composter

Se : moi je fais attention à l'eau.../...

$\mathrm{J}$ : récupération d'eau

Se : je peux pas j'ai même pas de balcon (rire)

Ainsi, le thème " empêchement d'agir » (tableau 2) renvoie en France à l'idée de contraintes externes au sujet (attributions externes): ici, c'est le fait d'habiter en ville qui empêche de récupérer l'eau de pluie ou de faire du compost, ailleurs ce sont les lois de la société qui interdisent à son producteur de jus de fruit de reprendre les bouteilles en verre... Ces contraintes externes se résument bien dans l'extrait ci-dessous, où les participants évoquent les voyages en avion :

\section{FG F2}

Cé : enfin c'est ce qu'on disait aussi sur la culpabilité quand comment tu réussis aussi après à vivre, et à te dire bon beh si je dois prendre l'avion pour mon boulot pour aller en Finlande par exemple au hasard, beh j'y vais bon je sais que je fais une connerie mais il faut aussi il faut aussi vivre on peut pas se couper non plus de tout ce qui est moi j'ai pas 
forcément envie de revenir euh, y'a 150 ans en arrière enfin pour que moi je m'occupe des gamins à la maison et que

.../...

Ch : mais du coup on en revient au changement de société comme tu disais aller bosser en Finlande bon t'as pas le choix tu peux pas prendre I'avion, voilà faudrait faire une société où t'as pas besoin d'être en Finlande et revenir trois jours après

Cé : ah oui il faudrait pouvoir mettre 15 jours pour y aller

Le voyage en Finlande pour le travail est considéré comme une contrainte imposée par la société et c'est ainsi que le groupe envisage un changement de société comme solution. La contrainte externe qui empêche ces sujets d'agir de manière écologique se distingue bien des intérêts pour soi avancés dans les groupes allemands (attribution interne : confort, besoin, etc.). Toutefois, cet extrait montre aussi que la notion de culpabilité est présente : en prenant I'avion on est infidèle à ses valeurs. Et c'est justement dans cette tension entre valeur individuelle et système social que prennent sens les pratiques écologiques en France.

Un autre élément intéressant est davantage lié aux dynamiques des discussions. Contrairement aux groupes allemands, il n'y a pas de débat autour d'action particulière. Les sujets français utilisent, en revanche, I'énumération de pratiques individuelles qui renvoient à une pratique supra-ordonnée acceptée de tous. Dans un des groupes, chaque participant explique par exemple dans le menu détail par quels moyens il économise de l'eau : des chasses d'eau moins gourmandes, un pommeau de douche spécial, etc. Cet aspect de la discussion montre que ce qui importe n'est donc pas tant l'efficacité réelle mais la valeur affichée par la pratique, en ce sens elles deviennent des pratiques symboliques. Ainsi, le « compromis écologique » trouve un sens particulier :

\section{FG F3}

G : je sais pas par exemple j'ai une copine qui fait vachement moi j'appelle ça cul pincée, ça m'énerve qui mange bio qui va jamais donner de bonbons à ses enfants, ma petite chérie tout ça c'est des cochonneries oui mais je les aime bien maman ces cochonneries (ils rient) donc oui donne des fraises tagada aux gamins, ça nous empêche pas d'être super logiques à plein de moments.

Les valeurs défendues par ces pratiques sont diverses, et parfois contradictoires (comme par exemple favoriser un fonctionnement collectif et être autonome). Comme l'indique les thèmes que nous avons cités (tableau 2), il s'agit de repenser ses besoins, de prendre le temps, de se satisfaire de peu. Un des groupes résume les pratiques écologiques à la fin de la discussion par le terme de "sobriété ».

\section{FG F2}

Ro : on peut mettre efficace partout à partir du moment où c'est bien fait c'est tout efficace quoi

Cé : en fait ça revient tout à réfléchir à ce qu'on fait, enfin c'est c'est prendre un peu de recul plutôt que de gober tout ce qu'on essaie de nous dire $c^{\prime}$ est de se dire

Ro : alors peut-être qu'on pourrait se dire pour garder cette grille là on pourrait se dire si on devait changer un truc dans la vie lequel on changerait en premier pour que ce soit 
vraiment efficace pour qu'on ait vraiment un gros gain... un gros gain de protection de l'environnement... et comme en plus on a des concepts qui sont

$\mathrm{E}$ : beh moi je mettrai sobriété

Ch : il englobe tout il en découle tout

$E$ : mais après $c^{\prime}$ est ça reste enfin $c^{\prime}$ est, enfin $y^{\prime}$ a des applications très pratiques mais ça reste une idée quoi

Les pratiques écologiques deviennent donc ici symbole d'une idée, d'une valeur plus générale que le sujet cherche à défendre. Les applications sont diverses, et les compromis possibles. En conséquence, en France, les pratiques écologiques viennent s'inscrire dans une tension entre l'expression de ses valeurs personnelles (forme de liberté) et une soumission aux contraintes imposées par la société (sorte d'obligation). La société est alors un Autre, différent du soi, qui empêche d'agir selon ses valeurs. Les entretiens individuels menés en parallèle montrent que ces valeurs ont une forte valeur politique, comme le souligne aussi l'extrait ci-dessous qui traite de l'achat direct aux producteurs plutôt qu'en supermarché :

\section{FG F3}

Se : on est d'accord je crois absolument raccourcir le circuit de distribution

$\mathrm{G}$ : alors après $\mathrm{c}^{\prime}$ est toujours pareil hein ça dépend de quel point de vue... parce que autant je pense que c'est efficace autant je pense que c'est complètement inefficace mais jouissif (ils rient)

$\mathrm{J}$ : dans les deux cas on se fait du bien

$\mathrm{G}$ : parce que ça emmerde les gros donc ça c'est

Se : ça c'est bien ouais

G : ça c'est le côté jouissif, parce que en plus euh on a l'impression de faire quelque chose de cohérent dans sa vie pour peu qu'on ait des convictions donc ça c'est génial

\subsection{Synthèse : pour penser les pratiques écologiques en France et en Allemagne}

La sphère des motifs de l'action proposée par Caillé (2009) nous permet de synthétiser et d'interpréter la différence France/Allemagne que nous avons mise en évidence dans nos résultats ( $c f$. Figure 1, ci-contre).

L'axe allemand figure en grisé, l'axe français en blanc. Ces motifs fonctionnent tous de manière dialogique. Néanmoins nos résultats indiquent que Français et Allemands ont tendance à inscrire leurs pratiques dans des catégories de pensée et dans des tensions différentes : liberté/obligation ; empathie/intérêt pour soi. Ces tensions structurent le discours sur les pratiques. En ce sens, elles renvoient à la notion de thêmata, définie comme " un mouvement de structuration d'un champ de connaissances ordinaires, communes et de significations partagées » (Moscovici et Vignaux, 1994, p. 64). Les thêmata que nous avons dégagés lors de notre analyse trouvent donc un appui théorique complémentaire dans les réflexions sociologiques d'inspiration maussienne (Caillé, 2009).

La façon dont Français et Allemands donnent sens à leurs pratiques met en jeu également un Alter différent. En Allemagne, la tension entre les motifs égoïste et empa- 
Figure 1 : Différents motifs de l'action en France et en Allemagne (d'après Caillé, 2009)

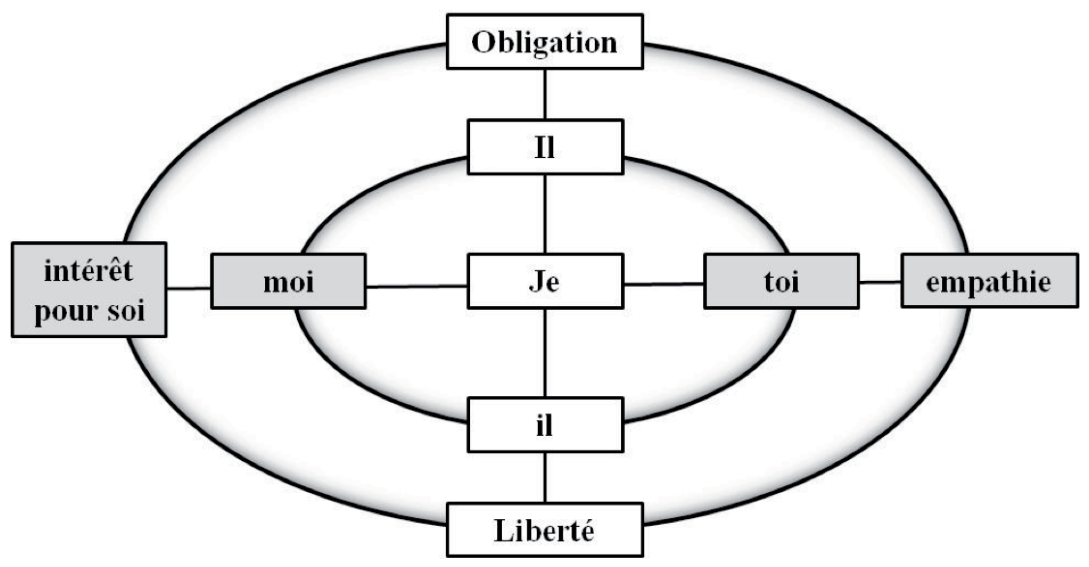

thique met en jeu un Alter-ego dans la manière dont on pense l'écologie. En France, $\mathrm{c}^{\prime}$ est au contraire un Alter, un système social dans lequel on ne se reconnait pas.

\section{Conclusions}

Nous nous sommes interrogés dans cet article sur la crise écologique et cela a été l'occasion de soulever un certain nombre de questions tant théoriques qu'empiriques. En premier lieu, nous avons montré l'existence d'une proximité entre l'approche des représentations sociales et l'idée d'une histoire humaine de la nature, telle qu'on la retrouve dans l'œuvre de Serge Moscovici. Rapprocher ces conceptualisations permet d'aborder la crise écologique comme un objet socialement construit, en dehors du dualisme nature/culture. L'usage des focus groups s'est révélé pertinent pour l'étude de la crise écologique, objet de communications interpersonnelles variées, mais également de pratiques individuelles qui prennent sens collectivement.

De plus, l'approche proposée ici nous a conduits à envisager les pratiques écologiques comme des actions poursuivant des motifs particuliers et faisant partie des représentations sociales. À la lumière de l'approche proposée et de nos résultats, deux conclusions essentielles peuvent être faites. Premièrement, on a pu constater que les pratiques écologiques, tant en France qu'en Allemagne, ne renvoient pas nécessairement à une volonté de protéger la nature et l'environnement et ne sont pas en premier lieu une réponse aux risques. Ces résultats, qui mériteraient d'être complétés par d'autres méthodes, invitent néanmoins à nuancer l'idée qu'en changeant les attitudes envers l'environnement, on change les comportements. Les pratiques deviennent alors en ce sens des pratiques signifiantes (Jodelet, 1989), renvoyant à des motifs moraux (en Allemagne) ou à une volonté d'exprimer des valeurs politiques personnelles (en France), motifs pour lesquels le support des autres semble toutefois essentiel. 
Cela nous amène à notre seconde remarque. Par l'étude des intentions liées aux actions écologiques, nous avons pu dégager que la crise écologique vient s'ancrer en France et en Allemagne dans des catégories de pensée différentes, fonctionnant sur un mode dialogique, et renvoyant ainsi à la notion de thêmata. La dimension morale que nous avons pu mettre en évidence en Allemagne vient faire écho à l'ancrage éthique du mouvement Vert allemand évoqué précédemment. En France, le caractère symbolique accordé aux pratiques, considérées comme une manière d'afficher des valeurs, fait écho à l'ancrage politique du mouvement Vert français. Mais celui-ci s'est également constitué autour d'associations naturalistes considérant la Science comme le référent. Cette dimension scientifique n'est pas plus présente en France qu'en Allemagne. Ce qui sert de référent, ce sont ici les pratiques des autres participants. On peut voir, dans le choix de ce référent, une manière de faire face au paradoxe des pratiques écologiques (des actions locales pour une crise globale).

Aussi, une crise majeure, celle de l'écologie, dont l'apparence est globale, mobilise en France et en Allemagne des thêmata différents (moral/immoral et liberté/obligation), alors qu'il s'agit pourtant de pays assez similaires. Il ne s'agit donc pas de qualifier les uns de plus écolos que les autres, mais bien de considérer qu'au sein de ces deux pays existent des lectures et a fortiori des interprétations différentes de la crise écologique.

La crise écologique apparaît ainsi dans notre étude comme un vaste système représentationnel, dépendant nécessairement du contexte socioculturel dans lequel il émerge et évolue. On parle de nature certes, mais pas seulement, et l'étude de ce système représentationnel nous apprend un certain nombre de choses sur notre rapport plus général au monde. S'intéresser aux représentations sociales de la crise écologique nous a également permis de prendre en considération les rapports que les sujets entretiennent avec leur nature, à travers des pratiques étiquetées écologiques, mais qui remplissent des fonctions multiples. Ainsi, la nature n'apparaît pas seulement comme un objet avec lequel on entretient des rapports, elle fait partie des sujets, les limites entre l'intérieur et l'extérieur ne se calquent pas sur un dualisme nature/culture, et cela fait de la nature un objet complexe. Mais accepter cette complexité, inhérente sans doute à un objet en crise, donc en mouvement, c'est aussi accepter que les risques écologiques représentent une menace pour l'altérité des sujets et des sociétés. 


\section{Notes}

1. Cette triade a d'ailleurs pour mérite de rappeler que le rapport de l'Homme à la nature dit aussi quelque chose du rapport des Hommes entre eux (Delbos et Jorion, 1988 ; Acot, 1998, Moscovici, 2002).

2. Ce modèle a par la suite été repris et complexifié sous la forme d'une rose des vents par Bauer et Gaskell (2008). Mais le modèle du Toblerone est suffisant pour saisir le sens de notre propos.

3. L'approche des RS présente un certain nombre d'autres avantages pour l'étude du rapport à l'environnement et à la nature comme l'a souligné, entre autres, Castro (2006).

4. En Allemagne de l'Est, les associations restent très surveillées par l'Etat.

5. À cet égard, la comparaison entre le Jardin du Luxembourg à Paris et du Tiergarten à Berlin montre l'influence des courants philosophiques.

6. http://ec.europa.eu/environment/pubs/pdf/brochure_fr.pdf (consulté le 13/07/2010).

7. http://ec.europa.eu/environment/archives/barometer/pdf/summary_ebenv_2005_04_22_fr.pdf (consulté le 13/07/2010).

8. La consigne qui leur était donnée : «De manière générale, quelles sont les actions que vous faites au quotidien et qui contribuent selon vous à la protection de la nature ? Je vous propose de faire une liste et d'essayer de définir en groupe dans quelle mesure chaque comportement est très efficace, efficace, peu efficace ou totalement inefficace, et pourquoi ? (Proposer un tableau que le groupe peut remplir). Vous n'êtes pas obligé de tomber d'accord à chaque fois, ce qui compte c'est davantage la discussion. »Cette consigne met bien l'accent sur l'importance du débat, afin de décentrer le groupe des seuls aspects relationnels de la discussion.

9. Ce critère n'est pas déterminant dans la mesure où les groupes déjà constitués n'entretiennent pas un rapport particulier à notre objet. En effet, il n'y a pas de groupe de militants écologistes par exemple. De plus, la thématique de discussion ne renvoie pas à la sphère intime, elle fait partie de ces sujets dont on parle dans des contextes sociaux assez variés. Notre analyse a tenu compte de cette variable, mais n'en a pas démontré des effets.

10. Le critère ex-Allemagne de I'Est et ex-Allemagne de l'Ouest ne peut être pris en compte ici. Toutefois, les groupes étaient hétérogènes en Allemagne, dans la mesure où cela correspond à la réalité actuelle du pays.

11. Voir tableau 2.

12. Le sigle FG D renvoie aux focus groups réalisés avec des Allemands, le sigle FG F à ceux réalisés avec des Français. De même, le chiffre juxtaposé permet de distinguer les différents groupes.

13. En Allemagne, le tri du verre se fait selon la couleur du verre et des containers à trois compartiments sont prévus à cet effet. 


\section{Bibliographie}

- Abric, J.-C. (2003). Les représentations sociales : aspects théoriques. In J.C. Abric (Ed.), Pratiques sociales et représentations (pp. 11-36). Paris: Presses Universitaires de France.

- Acot, P. (1998). Ecologie humaine et idéologie écologiste. In F. Aubert, \& J.-P. Sylvestre (Eds), Écologie et société (pp. 15-24). Dijon: Educagri.

- Barbour, R. (2008). Doing focus groups. Londres: Sage.

- Barr, S. (2007). Factors influencing environmental attitudes and behaviors. A UK study of household waste management. Environment and Behavior, 39 (4), 435-473.

- Bauer, M., \& Gaskell, G. (1999). Towards a paradigm for research on social representations. Journal for the Theory of Social Behaviour, 29 (2), 163-186.

- Bauer, M., \& Gaskell, G. (2008). Social representations theory: a progressive research programme for social psychology. Journal for the Theory of Social Behaviour, 38 (4), 335-353.

- Beck, U. (2001). La société du risque. Sur la voie d'une autre modernité. Paris: Aubier.

- Bourdieu, P. (1994). Raisons pratiques. Sur la théorie de l'action. Paris: Seuil.

- Buckhout, R. (1972). Pollution and the psychologist: A call to action. Environment and the Social Sciences, 75-81.

- Caillé, A. (2009). Théorie anti-utilitariste de l'action. Fragments d'une sociologie générale. Paris: La Découverte.

- Castro, P. (2006). Applying social psychology to the study of environmental concern and environmental worldviews: Contributions from the social representations approach. Journal of Community and Applied Social Psychology, 16, 247-266.

- Chibret, R.-P. (1991). Les associations écologiques en France et en Allemagne. Une analyse culturelle de la mobilisation collective. Thèse de doctorat en sciences politiques. Université Paris I.

- Cranach, von M., Kalbermatten, U., Indermühle, K., \& Gugler, B. (1982). Goal-directed action. Londres: Academic Press.

- Crozier, M., \& Friedberg, E. (1977). L'acteur et le système. Paris: Le Seuil.

- Delbos, G., \& Jorion, P. (1988). La nature ou le réel forclos. In A. Cadoret (Ed.), Chasser le naturel (pp. 15-21). Paris: Éditions de l'École des Hautes Études en Sciences Sociales.

- Eder, K. (1988). Die Vergesellschaftung der Natur. Studien zur sozialen Evolution der praktischen Vernunft. Frankfurt-am-Main: Suhrkamp Taschenbuch Verlag.

- Eder, K. (2000). L'environnement et le discours écologique : le cas de I'Allemagne. In M. Abélès, L. Charles, \& H.-P. Jeudy (Eds), L'environnement en perspective (pp. 191-208). Paris: L'Harmattan.

- Faucheux, C. (1976). Cross-cultural research in experimental social psychology. European Journal of Social Psychology, 6(3), 269-322.

- Ferry, L. (1992). Le nouvel ordre écologique. L'arbre, l'animal et l'homme. Paris: Bernard Grasset.

- Flament, C. (1987). Pratiques et représentations sociales. In J.-L. Beauvois, R.-V. Joule, \& J.M. Monteil (Eds), Perspectives cognitives et conduites sociales I : théories implicites et conflits cognitifs (pp. 143-150). Cousset: Delval.

- Flick, U. (1992). Triangulation revisited: strategy of validation or alternative? Journal for the Theory of Social Behaviour, 22 (2), 175-197.

- Flick, U. (1996). Psychologie des technisierten Alltags. Soziale Konstruktion technischen

Wandels. Opladen: Westdeutscher Verlag. 
- Flick, U. (2008). Designing qualitative research. London: Sage

- Frank, D.-J. (1997). Science, nature and the globalization of the environment, 1870-1990. Social Forces, 76 (2), 409-435.

- Gervais, M.-C. (1997). Social representations of nature. The case of the braer oil spill in Shetland. PhD on social psychology. London School of Economics and Political Sciences: University of London.

- Haas, V., \& Jodelet, D. (1999). Pensée et mémoire sociales. In J. P. Pétard (Ed.), Psychologie sociale (pp. 111-140). Paris: Éditions Bréal.

- Jacquiot, P. (2007). Comparaison des processus de formation et de diffusion du mouvement écologiste en RFA et en France. Cahiers Internationaux de Sociologie, 73, 217-244.

- Jacquiot, P. (2008). Mémoire collective du nazisme et engagement écologiste en ex-RFA. Nouvelle Revue de Psychosociologie, 5, 169-181.

- Jodelet, D. (1989). Folies et représentations sociales. Paris: Presses Universitaires de France.

- Jodelet, D. (2002). Les représentations sociales dans le champ de la culture. Information sur les Sciences Sociales, 41 (1), 111-132.

- Jodelet, D. (2006). Culture et pratiques de santé. Nouvelle Revue de Psychosociologie, 1, 219-239.

- Kalampalikis, N. (2002). Des noms et des représentations. Cahiers Internationaux de Psychologie Sociale, 53, 20-31.

- Kalampalikis, N. (2004). Les focus groups, lieux d'ancrages. Bulletin de Psychologie, 57 (3), 281-289.

- Kalampalikis, N. (2007). Les Grecs et le mythe d'Alexandre. Etude psychosociale d'un conflit symbolique à propos de la Macédoine. Paris: L'Harmattan.

- Kalaora, B. (1998). Au-delà de la nature l'environnement. L'observation sociale de l'environnement. Paris: L'Harmattan.

- Keller, R. (1998). Müll - die gesellschaftliche Konstruktion des Wertvollen. Die öffentliche Diskussion über Abfall in Deutschland und in Frankreich. Opladen: Westdeutscher Verlag.

- Kitzinger, J. (1994). The methodology of focus groups: the importance of interaction between research participants. Sociology of Health and Illness, 16 (1), 103-121.

- Kitzinger, J., Markova, I., \& Kalampalikis, N. (2004). Qu'est-ce que les focus groups ? Bulletin de Psychologie, 57 (3), 237-243.

- Kurz, T., Linden, M., \& Sheehy, N. (2007). Attitudinal and community influences on participation in new curbside recycling initiatives in Northern Ireland. Environment and Behavior, 39 (3), 367-391.

- Lenoble, R. (1969). Histoire de l'idée de nature. Paris: Albin Michel.

- Léont'ev, A. A. (1977). Tätigkeit, Bewusstsein, Persönnlichkeit. Stuttgart: Ernst Klett Verlag.

- Markova, I. (2003). Les focus groups. In S. Moscovici, \& F. Buschini (Eds), Les méthodes des sciences humaines (pp. 221-242). Paris: Presses Universitaires de France.

- Markova, I. (2007). Dialogicité et représentations sociales. Paris: Presses Universitaires de France.

- Matheau-Police, A., \& Moser, G. (2002). L'elasticité des conduites de tri sélectif. Recherche expérimentale : le comportement de tri des journaux et magazines des Parisiens du 14ème arrondissement. Cahiers Internationaux de Psychologie Sociale, 53, 79-88.

- Morgan, D. (1996). Focus groups. Annual Review of Sociology, 22, 129-152. 
- Moscovici, S. (1961). La psychanalyse, son image et son public. Paris: Presses Universitaires de France.

- Moscovici, S. (1968). Essai sur l'histoire humaine de la nature. Paris: Flammarion.

- Moscovici, S. (1974). Hommes domestiques et Hommes sauvages. Paris: Union Générale d'Éditions.

- Moscovici, S. (1989). Préface. In D. Jodelet (Ed.), Folies et représentations sociales. Paris: Presses Universitaires de France.

- Moscovici, S. (2001). Why a theory of social representations? In K. Deaux, \& G. Philogène (Eds), Representations of the Social (pp. 8-36). Oxford: Blackwell.

- Moscovici, S., \& Vignaux, G. (1994). Le concept de thêmata. In C. Guimelli (Ed.), Structures et transformations des représentations sociales (pp. 25-72). Neuchatel: Delachaux et Niestlé.

- Olli, E., Grendstad, G., \& Wollebaek, D. (2001). Correlates of environmental behaviors. Bringing back social context. Environment and Behavior, 33 (2), 181-208.

- Ollitrault, S. (2001). Les écologistes français, des experts en action. Revue Française de Sciences Politiques, 51 (1), 105-130.

- Rouquette, M.-L. (2000). Paradoxes de la représentation et de I'action: des conjonctions sans coordination. Revue Internationale des Sciences de l'Education, 4, 17-24.

- Rudolf, F. (1998). L'environnement, une construction sociale. Pratiques et discours sur l'environnement en France et en Allemagne. Strasbourg: Presses Universitaires de Strasbourg.

- Rudolf, F. (2005). Economie et écologie : les politiques environnementales de l'Allemagne contemporaine. In C. Demesnay, \& H. Stark (Eds), Qui dirige l'Allemagne? (pp. 213-225). Villeneuve d'Asq: Presses Universitaires du Septentrion.

- Schuster, K. (2003). Lebenstil und Akzeptanz von Naturschutz. Heidelberg und Kröning: Asanger Verlag.

- Stewart, D.W., Shamdasani, P.N., \& Rook, D.W. (2007). Focus Groups. Londres: Sage.

- Thogersen, J., \& Ölander, F. (2006). To what degree are environmentally beneficial choices reflective of a general conservation stance? Environment and Behavior, 38 (4), 550-569.

- Vigour, C. (2005). La comparaison dans les sciences sociales. Pratiques et méthodes. Paris: Editions La Découverte.

- Wagner, W. (1994). The fallacy of misplaced intentionality in social representation research. Journal for the Theory of Social Behaviour, 24 (3), 243-265. 